\title{
Defining Boot Camp: A Supporting Literature Review
}

\author{
Berridge, C. ${ }^{1}$, Jain, $S .{ }^{2}$ \& Biyani, C.S. ${ }^{2}$
}

\begin{abstract}
Introduction: Medical education boot camps have been shown to be effective courses to improve skills and confidence although the definition of what a boot camp is and how it differs from a skills course is not clear. We propose the definition of a boot camp to be an intensive course delivered at the start of a new clinical role designed to provide foundation to a curriculum through practical training.
\end{abstract}

Method: PubMed search using term "Boot Camp" and "Boot camp" with abstracts of results reviewed to determine if courses were at a transition in training role and if a curriculum was referred to.

Results: There were 109 relevant abstracts referring to boot camp courses. $81 \%$ referred to a curriculum and $74 \%$ of courses occurred at a transition in training roles. Half of all the abstracts were from surgical or surgical subspecialty curricula.

Conclusion: Our definition of a medical education boot camp is supported by the literature review performed. We hope that by defining boot camps it will encourage others to develop curriculum specific courses to support their trainees as they develop and enter new roles.

Key words: Simulation, Boot camp, Course, Surgery, Medical

\section{Introduction}

Medical education boot camps have embraced simulation training in order to provide 'handson' training in a controlled environment. The need for intensive courses covering a wide range of topics from a curriculum comes from increasingly complex management with decreasing opportunities for junior clinicians to gain experience and practice procedural skills (Milburn et al., 2012; Mittal, 2015). Boot camps have been shown to be effective in improving skills and confidence in applying knowledge and skills (Blackmore et al., 2014; Lerner et al., 2018; Kenny et al., 2018; Karmali et al., 2018). In addition, these courses still provide a significant increase to confidence for at least 6 months (Selden et al., 2013).

\footnotetext{
'Leicester General Hospital, United Kingdom

${ }^{2}$ St James's University Hospital, United Kingdom

Corresponding Author:

Chandra Shekhar Biyani

Consultant Urologist \& Hon Senior Lecturer, Department of Urology, St James's University Hospital, Leeds Teaching Hospitals NHS Trust, Beckett Street, Leeds, LS9 7TF.

Email: shekharbiyani@hotmail.com
}

Whilst these courses have been shown to be an effective way of introducing and refining skills and knowledge there is no clear definition that separates a boot camp from any other course. Blackmore et al. defined medical education boot camps as "courses designed to enhance learning and preparation for those entering new clinical roles with simulation-based practice and other related educational strategies" (Blackmore et al., 2014). We feel that a defined curriculum is also necessary from which a boot camp can be distilled to provide training on entering a new clinical role. A boot camp with this definition is truly different to a skills course and should be recognized as such (Table 1).

Our proposed definition of a medical education boot camp is an intensive course delivered at the start of a new clinical role designed to provide foundation to a curriculum through practical training.

In order to establish whether this definition is in keeping with previous boot camps a literature review was performed. 
Table 1: Differences between a boot camp and a skills course

\begin{tabular}{ll}
\hline \multicolumn{1}{c}{ Boot camp } & \multicolumn{1}{c}{ Skills course } \\
\hline Mapped to syllabus & Particular skill \\
Intense & Moderate \\
Before starting a NEW role & Anytime during the training \\
Majority hands-on & Variable \\
\hline
\end{tabular}

\section{Methods}

A PubMed search was performed with the search terms "Boot camp" and "Bootcamp". The abstracts for each of the search results were analysed by a single reviewer to determine the specialty organising the course and whether there was reference to a curriculum or syllabus.
In addition it was noted whether the course participants were at a transition in training role and at what grade that was. Publications were excluded if there was no abstract or if the content of the abstract did not refer to a clinical course (Figure 1). If the course covered a curriculum beyond a single specialty they were classed as "non-specific".

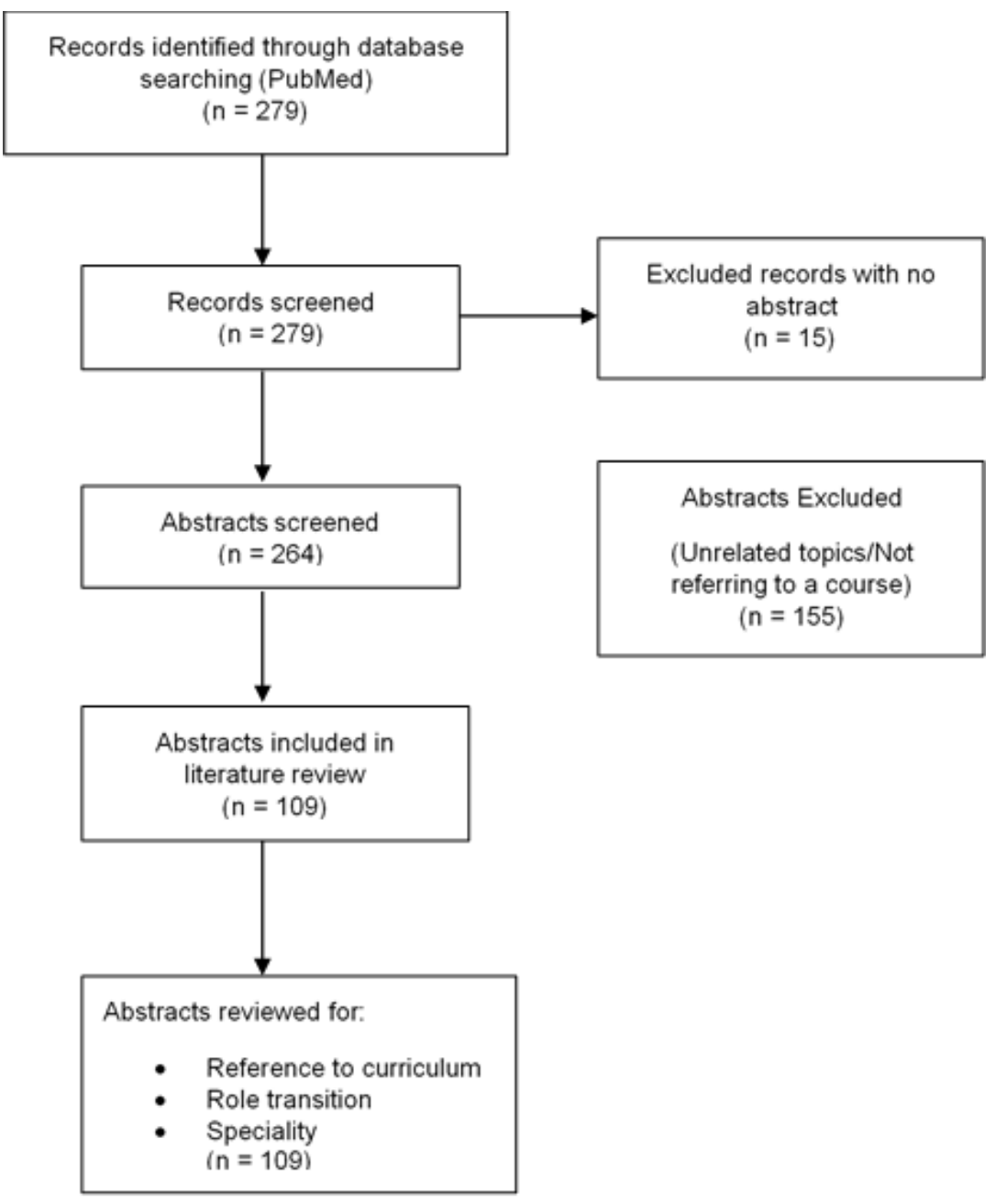

Figure 1: Literature review method displayed as PRISMA (Moher et al. 2009) flow chart 


\section{Results}

The PubMed search produced 279 results of which 109 were determined to be relevant (Table 2). The earliest paper was from 2006 with publications significantly increasing in number over the last 3 years (Figure 2). Twenty-two different specialties published regarding boot camps in addition to the nonspecific category. General surgery published the most $(15.6 \%)$ and half of all publications were from surgical specialties (50\%) (Figure 3 $\mathrm{a}, \mathrm{b})$. Medical specialties combined made up $9 \%$ of the relevant search results, which was at a similar level to that of emergency medicine, paediatrics and intensive care.

A curriculum was referred to in $81 \%$ of abstracts. The courses occurred at various points in training from medical school to fellows. Most commonly boot camps were for Residents or core trainees $(44 \%)$ at the beginning of specialist training. A quarter of courses were for newly qualified doctors (Intern or FY1) with 16\% of boot camps occurring whilst still at medical school. Overall $74 \%$ of boot camps took place at transition points in training when entering new clinical roles.

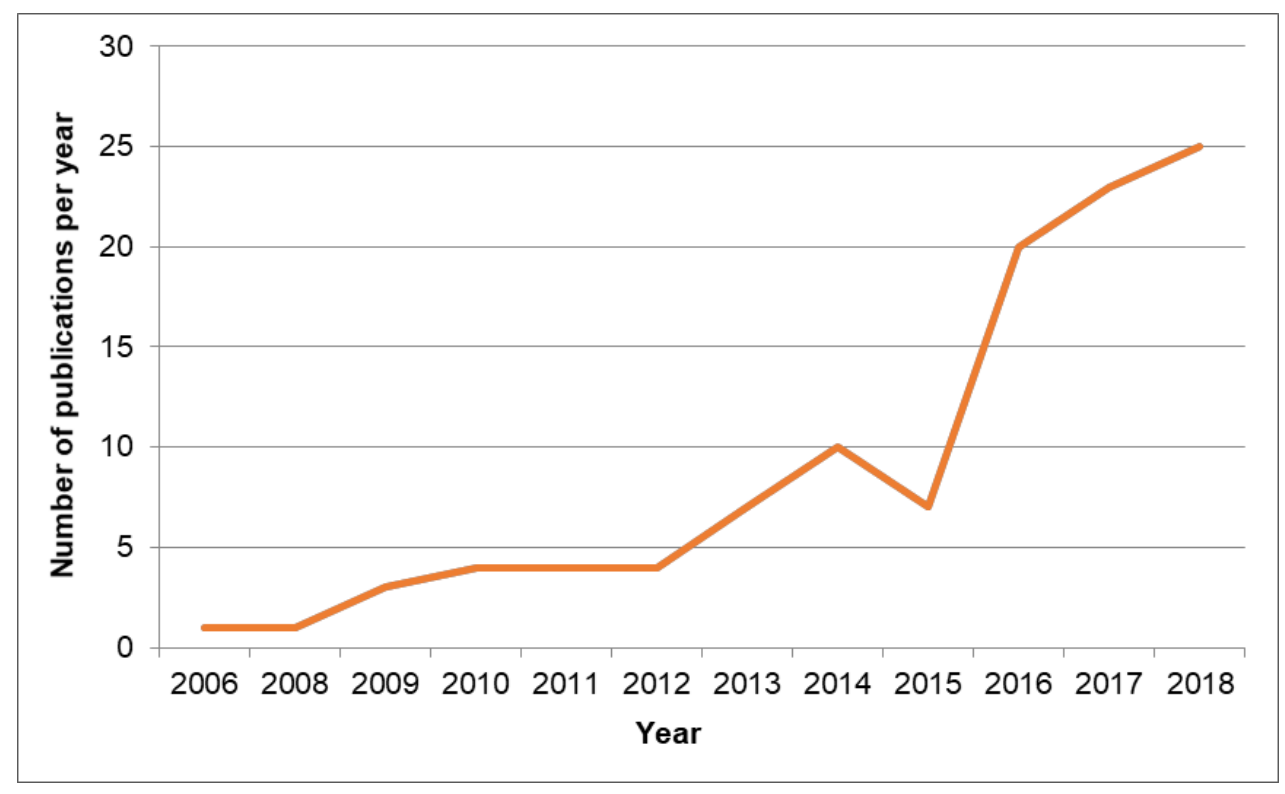

Figure 2: Number of publications per year referring to Boot camps
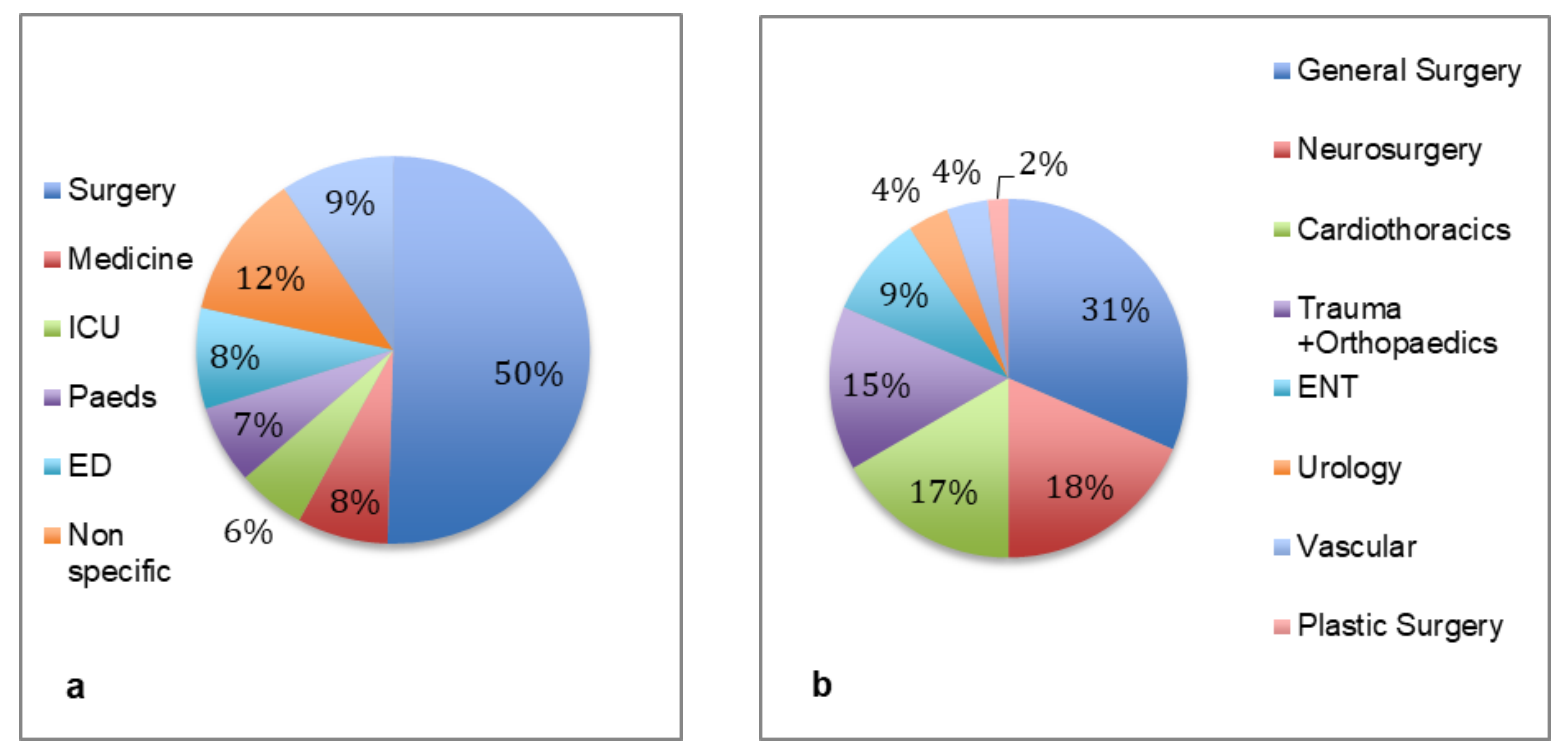

Figure $3(a \& b)$ : Number of publications per year referring to Boot camps 
Table 2: Summary of published abstracts detailing Boot camps.

\begin{tabular}{|c|c|c|c|c|}
\hline Specialty & $\begin{array}{c}\text { Total } \\
\text { Number } \\
\text { of } \\
\text { Abstracts }\end{array}$ & $\begin{array}{l}\text { Number } \\
\text { with } \\
\text { Curriculum }\end{array}$ & $\begin{array}{l}\text { Number } \\
\text { at } \\
\text { Transition } \\
\text { in grade }\end{array}$ & $\begin{array}{l}\text { Training grades with Boot } \\
\text { camps }\end{array}$ \\
\hline Surgery & 54 & 47 & 47 & $\begin{array}{l}\text { Student, Intern/FY1 Resident, ST3 } \\
\text { Fellow }\end{array}$ \\
\hline General Surgery & 17 & 15 & 16 & $\begin{array}{l}\text { Student, FY1/Intern, Resident, } \\
\text { Fellow }\end{array}$ \\
\hline Neurosurgery & 10 & 8 & 9 & Intern, Resident \\
\hline Cardiothoracic & 9 & 7 & 7 & Intern, Resident, ST3 \\
\hline $\begin{array}{l}\text { Trauma } \\
\text { +Orthopaedics }\end{array}$ & 8 & 8 & 7 & Student, FY1/Intern, Resident \\
\hline ENT & 5 & 4 & 4 & Resident \\
\hline Urology & 2 & 2 & 2 & ST3 \\
\hline Vascular & 2 & 2 & 1 & Fellow \\
\hline Plastic Surgery & 1 & 1 & 1 & Resident \\
\hline Medicine & 10 & 7 & 6 & Intern, Resident, Fellow \\
\hline ICU & 6 & 6 & 3 & Resident, Fellow \\
\hline Paediatrics & 7 & 7 & 4 & Student, FY1, Resident, Fellow \\
\hline ED & 9 & 5 & 5 & Student, Intern/FY1, Resident \\
\hline Non specific & 13 & 9 & 9 & Student, Intern, Resident \\
\hline Other & 10 & 6 & 6 & Student, Intern, Resident \\
\hline
\end{tabular}

Those with curricula identified and those that are specifically at a transition point in training are counted separately. Surgery is subdivided into sub-specialty. Grades listed if specifically referred to in published abstract ( $F Y 1=$ Foundation year 1 doctor - Intern equivalent, ST3= Specialty trainee - Senior resident equivalent)

\section{Discussion}

The 'Boot camp' has clearly become much more popular as a training experience over the last 3 years. This perhaps correlates with a growing understanding of the limitations of current training and methods to improve (Milburn et al., 2012). It is notable that specialties that have a curriculum dominated by procedural skills have published more abstracts related to boot camps. It is probable that surgical specialties lend themselves to the organising of boot camps with a multitude of procedural skills that can be introduced, critiqued and developed.
The proposed definition of medical education boot camps of an intensive course delivered at the start of a new clinical role designed to provide foundation to a curriculum through practical training is supported by the results of this literature search. With $81 \%$ of abstracts referring to a curriculum and $74 \%$ of courses being aimed at trainees at role transitions it is clear the majority of these courses would adhere to the proposed definition.

Courses were most commonly designed for residents or core trainees $(44 \%)$, which represents a significant stage of training when roles change from being led to being the leader. 


\section{Conclusion}

We have proposed a more specific definition of medical education boot camps in order to differentiate these from other skills courses. A literature search has clearly supported this definition and we hope that by defining boot camps it will encourage others to develop curriculum specific courses to support their trainees as they develop and enter new roles.

\section{References}

Milburn, J.A., Khera, G., Hornby, S.T., Malone, P.S.C. \& Fitzgerald, J.E.F. (2012) Introduction, availability and role of simulation in surgical education and training: review of current evidence and recommendations from the Association of Surgeons in Training.

Mittal, V.K. (2015) Simulation training-a necessity for future surgeons. Indian J Surg, 77, 4, 258-9

Blackmore, C., Austin, J., Lopushinsky, S.R. \& Donnon, T. (2014) Effects of postgraduate medical education "boot camps" on clinical skills, knowledge, and confidence: a meta-analysis. Journal of Graduate Medical Education, 6, 4, pp.643-652.
Lerner, V., Higgins, E.E. \& Winkel, A. (2018) Reboot: Simulation Elective for Medical Students as Preparation Bootcamp for Obstetrics and Gynecology Residency. Cureus, 10, 6.

Kenny, L., Booth, K., Freystaetter, K., Wood, G., Reynolds, G., Rathinam, S. \& Moorjani, N. (2018) Training cardiothoracic surgeons of the future: The UK experience. The Journal of Thoracic and Cardiovascular Surgery, 155, 6, pp.2526-2538.

Karmali, R.J., Siu, J.M., You, D.Z., Spano, S., Winthrop, A.L., Rudan, J.F., Reznick, R.K., Sanfilippo, A.T. \& Belliveau, P. (2018) The Surgical Skills and Technology Elective Program (SSTEP): A comprehensive simulation-based surgical skills initiative for preclerkship medical students. The American Journal of Surgery, 216, 2, pp.375-381.

Selden, N.R., Anderson, V.C., McCartney, S., Origitano, T.C., Burchiel, K.J. \& Barbaro, N.M. (2013) Society of Neurological Surgeons boot camp courses: knowledge retention and relevance of hands-on learning after 6 months of postgraduate year 1 training. Journal of Neurosurgery, 119, 3, pp.796-802.

Moher, D., Liberati, A., Tetzlaff, J. \& Altman, D.G. (2009) Preferred reporting items for systematic reviews and meta-analyses: the PRISMA statement. Annals of Internal Medicine, 151, 4, pp.264-269. 


\section{Appendix:}

\section{Abstracts Reviewed}

1. Acosta, D., Castillo-Angeles, M., GarcesDescovich, A., Watkins, A.A., Gupta, A., Critchlow, J.F., Kent, T.S. (2018). Surgical Practical Skills Learning Curriculum: Implementation and Interns' Confidence Perceptions, J Surg Educ, 75, 2, 263-270.

2. Al Achkar, M., Davies, M.K. (2016). A small group learning model for evidence-based medicine, Adv Med Educ Pract, 7, pp. 611-615.

3. Allan, C.K., Tannous, P., DeWitt, E., Farias, M., Mansfield, L., Ronai, C., Schidlow, D., Sanders, S.P., Lock, J.E., Newburger, J.W., Brown, D.W. (2016). A Pediatric Cardiology Fellowship Boot Camp improves trainee confidence, Cardiol Young, 26, 8, pp. 1514-1521.

4. Ambardekar, A.P., Singh, D., Lockman, J.L., Rodgers, D.L., Hales, R.L., Gurnaney, H.G., Nathan, A., Deutsch, E.S. (2016). Pediatric anesthesiology fellow education: is a simulationbased boot camp feasible and valuable? Paediatr Anaesth, 26, 5, pp. 481-7.

5. Ament, J.D., Kim, T., Gold-Markel, J., Germano, I.M., Dempsey, R., Weaver, J.P., DiPatri, A.J Jr., Andrews, R.J., Sanchez, M., Hinojosa, J., Moser, R.P., Glick, R. (2017). Planning and Executing the Neurosurgery Boot Camp: The Bolivia Experience, World Neurosurg, 104, pp.407-410.

6. Ataya, R., Dasgupta, R., Blanda, R., Moftakhar, Y., Hughes, P.G., Ahmed, R. (2015). Emergency medicine residency boot Camp curriculum: a pilot study, West J Emerg Med, 16, 2, pp. 356-61.

7. Bagley, K.A., Dunn, S.E., Chuang, E.Y., Dorr, V.J., Thompson, J.A., Smith, S.K. (2018). Nonspecialty Nurse Education: Evaluation of the Oncology Intensives Initiative, an Oncology Curriculum to Improve Patient Care, Clin J Oncol Nurs, 22, 2, pp.E44-E51. doi: 10.1188/18.CJON.E44-E51.

8. Bamford, R., Langdon, L., Rodd, C.A., Eastaugh-Waring, S., Coulston, J.E. (2018). Core trainee boot camp, a method for improving technical and non-technical skills of novice surgical trainees. A before and after study, Int $\mathrm{J}$ Surg, 57, pp. 60-65.

9. Bierer, J., Memu, E., Leeper, W.R., Fortin, D., Frachette, E., Inculet, R., Malthaner, R. (2018). Development of an In Situ Thoracic Surgery Crisis Simulation Focused on Nontechnical Skill Training, Ann Thorac Surg, 106, 1, pp. 287-292.
10. Bismuth, J., Duran, C., Donovan, M., Davies, M.G., Lumsden, A.B. (2012). The Cardiovascular Fellows Bootcamp, J Vasc Surg, 56, 4, pp. 1155-61.

11. Bontempo, L.J., Frayha, N., Dittmar, P.C. (2017). The Internship Preparation Camp at the University of Maryland, Postgrad Med J, 93, 1095, pp. 8-14.

12. Brown, K.M., Mudd, S.S., Hunt, E.A., Perretta, J.S., Shilkofski, N.A., Diddle, J.W., Yurasek, G., Bembea, M.,Duval-Arnould, J., Nelson McMillan, K. (2018). A Multi-Institutional Simulation Boot Camp for Pediatric Cardiac Critical Care Nurse Practitioners, Pediatr Crit Care Med, 19,6, pp. 564-571.

13. Busing, N., Rosenfield, J., Rungta, K., Raegele, M., Warren, A., Wright, B., Walton, M., Oandasan, I., Sanfilippo, A., Saxena, A. (2018). Smoothing the Transition Points in Canadian Medical Education, Acad Med, 93, 5, pp.715 721.

14. Burns, R., Adler, M., Mangold, K., Trainor, J. (2016). A Brief Boot Camp for 4th-Year Medical Students Entering into Pediatric and Family Medicine Residencies, Cureus, 8, 2, pp. e488.

15. Carpenter, C.R., Melady, D., Krausz, C., Wagner, J., Froelke, B., Cordia, J., Lowery, D., Ruoff, B.E., Byrne, L.E., Miller, D.K., Lewis, L. (2017). Improving Emergency Department Care for Aging Missourians: Guidelines, Accreditation, and Collaboration, Mo Med, 114, 6, pp.447-452.

16. Carter, Y.M., Wilson, B.M., Hall, E., Marshall, M.B. (2010). Multipurpose simulator for technical skill development in thoracic surgery, J Surg Res, 163, 2, pp. 186-91.

17. Castillo, J., Kutzin, J., Agoglia, K., Janicke, P., Milligan, Z., Scott, J. (2017). An Innovative Clinical Skills "Boot Camp" for Dental Medicine Residents. J Med Educ Curric Dev. May 29; eCollection.

18. Ceresnak, S.R., Axelrod, D.M., Sacks, L.D., Motonaga, K.S., Johnson, E.R., Krawczeski, C.D. (2017). Advances in Pediatric Cardiology Boot Camp: Boot Camp Training Promotes Fellowship Readiness and Enables Retention of Knowledge, Pediatr Cardiol, 38, 3, pp. 631-640.

19. Ceresnak, S.R., Axelrod, D.M., Motonaga, K.S., Johnson, E.R., Krawczeski, C.D. (2016). Pediatric Cardiology Boot Camp: Description and Evaluation of a Novel Intensive Training Program for Pediatric Cardiology Trainees, Pediatr Cardiol, 37, 5, pp. 834-44. 
20. Chin, C.J., Roth, K., Rotenberg, B.W., Fung, K. (2014). Emergencies in otolaryngology-head and neck surgery bootcamp: a novel Canadian experience, Laryngoscope, 124,10, pp. 227580.

21. Chin, C.J., Chin, C.A., Roth, K., Rotenberg, B.W., Fung, K. (2016). Simulation-based otolaryngology - head and neck surgery boot camp: 'how I do it', J Laryngol Otol, 130, 3, pp. 284-90.

22. Cleary, D.R., Siler, D.A., Whitney, N., Selden, N.R. (2018). A microcontroller-based simulation of dural venous sinus injury for neurosurgical training, J Neurosurg, 128, 5, pp. 1553-1559.

23. Cohen, E.R., Barsuk, J.H., Moazed, F., Caprio, T., Didwania, A., McGaghie, W.C., Wayne, D.B. (2013). Making July safer: simulation-based mastery learning during intern boot camp, Acad Med, 88, 2, pp. 233-9.

24. Crews, C.S., Minor, L.C. (2018). Evaluation and Student Perceptions of an OB Boot Camp Simulation for Undergraduate Nursing Students: A Mixed-Method Approach, Nurs Educ Perspect, 39,1, pp. 35-37.

25. Davidson, E.H., Barker, J.C., Egro, F.M., Krajewski, A., Janis, J.E., Nguyen, V.T. (2017). A National Curriculum of Fundamental Skills for Plastic Surgery Residency: Report of the Inaugural ACAPS Boot Camp, Ann Plast Surg, 78, 2, pp. 121-126.

26. Derman, P.B., lyer, S., Garner, M., Orr, S., Felix, K.J., Goldberg, A., Ologhobo, T., Wu, M., Robbins, L., Cornell, C. (2016). An Initiative to Standardize the Identification of and Acute Response to Postoperative Lower-Extremity Neurological Deficits: Effects on Provider Knowledge, Confidence, and Communication Skills, J Bone Joint Surg Am, 98, 23, p. e105.

27. Devon, E.P., Tenney-Soeiro, R., Ronan, J., Balmer, D.F. (2018). A Pediatric Pre-Intern Boot Camp: Program Development and Evaluation informed by a Conceptual Framework, Acad Pediatr, S1876-2859, 18, pp.30535-7.

28. Duncanson, K., Webster, E.L., Schmidt, D.D. (2018). Impact of a remotely delivered, writing for publication program on publication outcomes of novice researchers, Rural Remote Health, 18, 2, p. 4468. doi: 10.22605/RRH4468.

29. Duran, C.A., Shames, M., Bismuth, J., Lee, J.T., Simulation Committee of the Association of Program Directors inVascular Surgery. (2014). Validated assessment tool paves the way for standardized evaluation of trainees on anastomotic models, Ann Vasc Surg, 28, 1, pp. $115-21$
30. Esch, L.M., Bird, A.N., Oyler, J.L., Lee, W.W., Shah, S.D., Pincavage, A.T. (2015). Preparing for the primary care clinic: an ambulatory boot camp for internal medicine interns, Med Educ Online, 20, p. 29702.

31. Esterl, R.MJr., Henzi, D.L., Cohn, S.M. (2006). Senior medical student "Boot Camp": can result in increased self-confidence before starting surgery internships, Curr Surg, 63, 4, pp. 264-8.

32. Fann, J.I., Calhoon, J.H., Carpenter, A.J., Merrill, W.H., Brown, J.W., Poston, R.S., Kalani, M., Murray, G.F., Hicks, G.L Jr., Feins, R.H. (2010). Simulation in coronary artery anastomosis early in cardiothoracic surgical residency training: the Boot Camp experience, $\mathrm{J}$ Thorac Cardiovasc Surg, 139,5, pp.1275-81.

33. Fernandez, G.L., Page, D.W., Coe, N.P., Lee, P.C., Patterson, L.A., Skylizard, L., St Louis, M., Amaral, M.H., Wait, R.B., Seymour, N.E. (2012). Boot CAMP: educational outcomes after 4 successive years of preparatory simulationbased training at onset of internship, J Surg Educ, 69, 2, 242-8.

34. Fontes, R.B., Selden, N.R., Byrne, R.W. (2014). Fostering and assessing professionalism and communication skills in neurosurgical education, J Surg Educ, 71, 6, pp. e83-9.

35. Green, C.A., Huang, E., Zhao, N.W., O'Sullivan, P.S., Kim, E., Chern, H. (2018). Technical skill improvement with surgical preparatory courses: What advantages are reflected in residency? Am J Surg, 216, 1, pp. 155-159.

36. Gradl, G., Bahren, A., Simon, M., Derntl, B., Pape, H.C., Knobe, M. (2017). Bootcamp: longitudinal gender based surgical and clinical skills training, Unfallchirurg, 120, 1, pp. 46-54.

37. Gunther, J.R., Jimenez, R.B., Yechieli, R.L., Parekh, A., Berman, A.T., Braunstein, S.E., Hirsch, A.E., Gillespie, E.F., Vapiwala, N., Thomas, C.RJr., Fields, E.C., Golden, D.W. (2018). Introductory Radiation Oncology Curriculum: Report of a National Needs Assessment and Multi-institutional Pilot Implementation, Int J Radiat Oncol Biol Phys, 101,5, pp. 1029-1038.

38. Haglin, J.M., Zeller, J.L., Egol, K.A., Phillips, D.P. (2017). Examination to assess the clinical examination and documentation of spine pathology among orthopedic residents, Spine J, 17,12 , pp.1830-1836.

39. Heimroth, J., Jones, V.M., Howard, J.DJr., Sutton, E.R.H. (2018). Utilizing Surgical Bootcamps to Teach Core Entrustable Professional Activities to Senior Medical Students, Am Surg, 84,6, pp. 783-788. 
40. Herling, P.J., Mohseni, B.T., Hill, D.C., Chelf, S., Rickert, J.A., Leo, J.T., Langley, N.R. (2017). Impact of Anatomy Boot Camp on Students in a Medical Gross Anatomy Course, Anat Sci Educ, 10, (3), pp. 215-223, doi: 10.1002/ase.1653.

41. Heskin, L., Mansour, E., Lane, B., Kavanagh, D., Dicker, P., Ryan, D., Gildea-Byrne, K., Pawlikowska, T., Tierney, S., Traynor, O. (2015). The impact of a surgical boot camp on early acquisition of technical and nontechnical skills by novice surgical trainees, Am J Surg, 210, 3, pp. 570-7.

42. Hicks, G.L Jr., Gangemi, J., Angona, R.E Jr., Ramphal, P.S., Feins, R.H., Fann, J.I. (2011). Cardiopulmonary bypass simulation at the Boot Camp, J Thorac Cardiovasc Surg, 141, 1, pp. 284-92.

43. Hogewood, C., Smith, T., Etheridge, S., Britt, S. (2015). Clinical Boot Camp: An Innovative Simulation Experience to Prepare Nursing Students for Obstetric and Pediatric Clinicals, Nurs Educ Perspect, 36, 6, pp. 410-1.

44. Hunt, M.A., Heilman, C.B., Shutran, M., Wu, J.K. (2017). Commentary: An Introduction to Leadership Self Assessment at the Society of Neurological Surgeons Post-Graduate Year 1 Boot Camp: Observations and Commentary, Neurosurgery, 80, 3, pp. E201-E204.

45. Jaswal, J., D'Souza, L., Johnson, M., Tay, K., Fung, K., Nichols, A., Landis, M., Leung, E., Kassam, Z., Willmore, K., D'Souza, D., Sexton, T., Palma, D.A. (2015). Evaluating the impact of a Canadian national anatomy and radiology contouring boot camp for radiation oncology residents, Int J Radiat Oncol Biol Phys, 91, 4, pp. 701-7.

46. Kailavasan, M., Hanchanale, V., Rajpal, S., Morley, R., Mcllhenny, C., Somani, B., Nabi, G., Gowda, R., Jain, S., Biyani, C.S., Myatt, A. (2019). A Method to Evaluate Trainee Progression During Simulation Training at the Urology Simulation Boot Camp (USBC) Course, J Surg Educ, 76, 1, pp. 215-222.

47. Karmali, R.J., Siu, J.M., You, D.Z., Spano, S., Winthrop, A.L., Rudan, J.F., Reznick, R.K., Sanfilippo, A.T., Belliveau, P. The Surgical Skills and Technology Elective Program (SSTEP) (2018): A comprehensive simulation-based surgical skills initiative for preclerkship medical students, Am J Surg, 216, 2, pp. 375-381.

48. Kenny, L., Booth, K., Freystaetter, K., Wood, G., Reynolds, G., Rathinam, S., Moorjani, N. (2018). Training cardiothoracic surgeons of the future: The UK experience, J Thorac Cardiovasc Surg, 155, 6, pp. 2526-2538.

49. Khobrani, A., Patel, N.H., George, R.L., McNinch, N.L., Ahmed, R.A. (2018). Pediatric Trauma Boot Camp: A Simulation Curriculum and Pilot Study, Emerg Med Int, 2018:7982315. doi: 10.1155/2018/7982315. eCollection 2018.

50. Kim, J.H., Kim, S.W., Kim, Y.M., Cho, Y., Lee, S.J. (2018). Effect of the curved blade size on the outcomes of tracheal intubation performed by incoming interns: A randomized controlled manikin study, Medicine (Baltimore), 97, 34, p.e11984.

51. Krajewski, A., Filippa, D., Staff, I., Singh, R., Kirton, O.C. (2013). Implementation of an intern boot camp curriculum to address clinical competencies under the new Accreditation Council for Graduate Medical Education supervision requirements and duty hour restrictions, JAMA Surg, 148, 8, pp. 727-32.

52. Kreshak, A.A., Neath, S.X., Tolia, V.M., Castillo, E.M., Chan, T.C. (2018). A Multidisciplinary Bootcamp as an Educational Launch to a Geriatric Emergency Department, J Emerg Med, 54, 6, pp. 855-860.

53. Laack, T.A., Newman, J.S., Goyal, D.G., Torsher, L.C. (2010). A 1-week simulated internship course helps prepare medical students for transition to residency, Simul Healthc, 5, 3, pp. 127-32.

54. Lamba, S., Wilson, B., Natal, B., Nagurka, R., Anana, M., Sule, H. (2016). A suggested emergency medicine boot camp curriculum for medical students based on the mapping of Core Entrustable Professional Activities to Emergency Medicine Level 1 milestones, Adv Med Educ Pract, 7, pp. 115-24.

55. Langdorf, M.I., Strom, S.L., Yang, L., Canales, C., Anderson, C.L., Amin, A., Lotfipour, S. (2014). High-fidelity simulation enhances ACLS training, Teach Learn Med, 26,3, pp.266-73.

56. Lee, E.H., Jun, J.K., Jung, S.E., Kim, Y.M., Choi, N. (2014). The efficacy of mammography boot camp to improve the performance of radiologists, Korean J Radiol 15, 5, pp. 578-85.

57. Lerner, V., Higgins, E.E., Winkel, A. (2018). Reboot: Simulation Elective for Medical Students as PreparationBootcamp for Obstetrics and Gynecology Residency, Cureus, 10, 6, p. e2811.

58. Levin, A.S., Haq, I.U., LaPorte, D.M. (2018). Pre-course cognitive training using a smartphone application in orthopaedic intern surgical skills "boot camps", J Orthop, 15, 2, pp. 506-508. 
59. Lou, X., Lee, R., Feins, R.H., Enter, D., Hicks, G.L Jr., Verrier, E.D., Fann, J.I. (2014). Training less-experienced faculty improves reliability of skills assessment in cardiac surgery, $\mathrm{J}$ Thorac Cardiovasc Surg, 148, 6, pp. 2491-6.

60. Macfie, R.C., Webel, A.D., Nesbitt, J.C., Fann, J.I., Hicks, G.L., Feins, R.H. (2014). Boot camp" simulator training in open hilar dissection in early cardiothoracic surgical residency, Ann Thorac Surg, 97,1, pp.161-6.

61. Malekzadeh, S., Malloy, K.M., Chu, E.E., Tompkins, J., Battista, A., Deutsch, E.S. (2011). ORL emergencies boot camp: using simulation to onboard residents, Laryngoscope, 121, 10, pp. 2114-21.

62. Malloy, K.M., Malekzadeh, S., Deutsch, E.S. (2014). Simulation-based otorhinolaryngology emergencies boot camp: Part 1: Curriculum design and airway skills, Laryngoscope, 124, 7, pp. 1562-5.

63. Maskatia, S.A., Altman, C.A., Morris, S.A., Cabrera, A.G. (2013). The echocardiography "boot camp": a novel approach in pediatric cardiovascular imaging education, J Am Soc Echocardiogr, 26, 10, pp. 1187-1192.

64. Maskatia, S.A., Cabrera, A.G., Morris, S.A., Altman, C.A. (2017). The pediatric echocardiography Boot Camp: Four-year experience and impact on clinical performance, Echocardiography, 34, 10, pp.1486-1494.

65. Min, A.A., Stoneking, L.R., Grall, K.H., SpearEllinwood, K. (2014). Implementation of the Introductory Clinician Development Series: an optional boot camp for Emergency Medicine interns, Adv Med Educ Pract, 25, 5, pp. 275-9.

66. Minha, S., Shefet, D., Sagi, D., Berkenstadt, H., Ziv, A. (2016). See One, Sim One, Do One"- A National Pre Internship Boot-Camp to Ensure a Safer "Student to Doctor" Transition, PLoS One, 11, 3, pp. e0150122.

67. Minkowitz, S., Leeman, K., Giambrone, A.E., Kherani, J.F., Belfi, L.M., Bartolotta, R.J. (2017). Emergency Radiology "Boot Camp": Educating Emergency Medicine Residents Using Elearning Radiology Modules, AEM Educ Train, 1, 1, pp. 43-47.

68. Moazed, F., Cohen, E.R., Furiasse, N., Singer, B., Corbridge, T.C., McGaghie, W.C., Wayne, D.B. (2013). Retention of critical care skills after simulation-based mastery learning, J Grad Med Educ, 5, 3, pp. 458-63.

69. Naritoku, W.Y., Vasovic, L., Steinberg, J.J., Prystowsky, M.B., Powell, S.Z. (2014). Anatomic and clinicalpathology boot camps: filling pathology-specific gaps in undergraduate medical education, Arch Pathol Lab Med,138, 3, pp. 316-21.

70. Nesbitt, J.C., Michaud, N.M., Brakebill, A., Deppen, S.A., Williams, P. (2018). Month-long cardiac surgery boot camp: A proposal to jumpstart resident training, J Thorac Cardiovasc Surg, 156, 3, pp. 1151-1157.

71. Nitsche, J.F., Fino, N.F., Palomo, J.M., Perdomo, A.P., Brost, B.C. (2017). Effectiveness of a Labor Cervical Exam Model in Family Medicine and OB-GYN Residents, Fam Med, 49, 5, pp. 384-387.

72. Nishisaki, A., Hales, R., Biagas, K., Cheifetz, I., Corriveau, C., Garber, N., Hunt, E., Jarrah, R., McCloskey, J., Morrison, W., Nelson, K., Niles, D., Smith, S., Thomas, S., Tuttle, S., Helfaer, M., Nadkarni, V. (2009). A multi-institutional highfidelity simulation "boot camp" orientation and training program for first year pediatric critical care fellows, Pediatr Crit Care Med, 10, 2, pp. 157-62.

73. Offiah, G., Doherty, E. (2018). Tricks of the trade: time management tips for newly qualified doctors, Postgrad Med, 94, 1109, pp. 159-161.

74. Omlor, R.L., Watkins, F.S., Lawlor, J.S., Lovato, J.F., Fino, N.F., Atkinson, H.H. (2017). Intern Boot Camp: Feasibility and impact of a 1-hour session to ensure graduating medical student competency in falls risk assessment, Gerontol Geriatr Educ, 38, 3, pp. 346-353.

75. Ortiz Figueroa, F., Moftakhar, Y., Dobbins Iv, A.L., Khan, R., Dasgupta, R., Blanda, R., Marchand, T., Ahmed, R. (2016). Trauma Boot Camp: A Simulation-Based Pilot Study, Cureu, 20, 8(1), p. e463. doi: 10.7759/cureus.463.

76. Parent, R.J., Plerhoples, T.A., Long, E.E., Zimmer, D.M., Teshome, M., Mohr, C.J., Ly, D.P., Hernandez, Boussard, T., Curet, M.J., Dutta, S. (2010). Early, intermediate, and late effects of a surgical skills "boot camp" on an objective structured assessment of technical skills: a randomized controlled study, J Am Coll Surg, 210, 6, pp. 984-9.

77. Pines, A.R., Alghoul, M.S., Hamade, Y.J., Sattur, M.G., Aoun, R.J.N., Halasa, T.K., Krishna, C., Zammar, S.G., El Tecle, N.E., El Ahmadieh, T.Y., Aoun, S.G., Byrne, R.W., Harrop, J.S., Ragel, B.T., Resnick, D.K., Lonser, R.R., Selden, N.R., Bendok, B.R. (2017). Assessment of the Interrater Reliability of the Congress of Neurological Surgeons Microanastomosis Assessment Scale, Oper Neurosurg (Hagerstown), 13(1), pp. 108-112. doi: 10.1227/NEU.0000000000001403. 
78. Pliego, J.F., Wehbe-Janek, H., Rajab, M.H., Browning, J.L., Fothergill, R.E. (2008). OB/GYN boot cAMP using high-fidelity human simulators: enhancing residents' perceived competency, confidence in taking a leadership role, and stress hardiness, Simul Healthc, 3, 2, pp. 82-9.

79. Ràjbago, J.L., Lopez-Doueil, M., Sancho, R., Hernàoojndez-Pinto, P., Neira, N., Capa, E., Larraz, E., Redondo Figuero, C.G., Maestre, J.M. (2017). Learning outcomes evaluation of a simulation-based introductory course to anaesthesia, Rev Esp Anestesiol Reanim, 64, 8, pp. 431-440.

80. Rock, J., Glick, R., Germano, I.M., Dempsey, R., Zervos, J., Prentiss, T., Davis, M., Wright, E., Hlaing, K., Thu, M., Soe, Z.W., Myaing, W. (2018). The First Neurosurgery Boot Camp in Southeast Asia: Evaluating Impact on Knowledge and Regional Collaboration in Yangon, Myanmar, World Neurosurg, 113, pp. e239-e246.

81. Ruff, I.M., Liberman, A.L., Caprio, F.Z., Maas, M.B., Mendelson, S.J., Sorond, F.A., Bergman, D., Bernstein, R.A., Curran, Y., Prabhakaran, S. (2017). A resident boot camp for reducing doorto-needle times at academic medical centers, Neurol Clin Pract, 7, 3, pp. 237-245.

82. Scherer, J.J., Reichrath, S., Tschernig, T., Maxeiner, S. (2019). A snapshot of anatomy teaching: Peer-led anatomy boot camps priming for success? Ann Anat, 221, pp.141147.

83. Schieman, C., Ujiie, H., Donahoe, L., Hanna, W., Malthaner, R., Turner, S., Czarnecka, K., Yasufuku, K. (2018). Developing a National, Simulation-Based, Surgical Skills Bootcamp in General Thoracic Surgery, J Surg Educ, 75, 4, pp. 1106-1112.

84. Schoolfield, C.S., Samra, N., Kim, R.H., Shi, R., Zhang, W.W., Tan, T.W. (2016). Evaluating the Effectiveness of the General Surgery Intern Boot Camp, Am Surg, 82, 3, pp. 243-50.

85. Schnobrich, D.J., Mathews, B.K., Trappey, B.E., Muthyala, B.K., Olson, A.P.J. (2018). Entrusting internal medicine residents to use point of care ultrasound: Towards improved assessment and supervision, Med Teach, 23, pp. 1-6, doi: 10.1080/0142159X.2018.1457210.

86. Scott, G.M., Fung, K., Roth, K.E. (2016). Novel High-Fidelity Peritonsillar Abscess Simulator, Otolaryngol Head Neck Surg, 154, 4, 634-7

87. Seddon, J. (2018). General practitioners teaching new surgical trainees about clinic letter writing, Clin Teach,15,1, pp. 44-47.

88. Seeley, M.A., Kazarian, E., King, B., Biermann, J.S., Carpenter, J.E., Caird, M.S., Irwin, T.A.
(2016). Core Concepts: Orthopedic Intern Curriculum Boot Camp, Orthopedics, 39,1, pp. e62-7.

89. Selden, N.R., Origitano, T.C., Burchiel, K.J., Getch, C.C., Anderson, V.C., McCartney, S., Abdulrauf, S.I., Barrow, D.L., Ehni, B.L., Grady, M.S., Hadjipanayis, C.G., Heilman, C.B., Popp, A.J., Sawaya, R., Schuster, J.M., Wu, J.K., Barbaro, N.M. (2012). A national fundamentals curriculum for neurosurgery PGY1 residents: the 2010 Society of Neurological Surgeons boot camp courses, Neurosurgery, 70, 4, pp. 971-81.

90. Selden, N.R., Barbaro, N., Origitano, T.C., Burchiel, K.J. (2011). Fundamental skills for entering neurosurgery residents: report of a Pacific region "boot camp" pilot course, 2009, Neurosurgery, 68, 3, pp. 759-64, discussion 764.

91. Selden, N.R., Origitano, T.C., Hadjipanayis, C., Byrne, R. (2013). Model-based simulation for early neurosurgical learners, Neurosurgery, 73, Suppl 1, pp.15-24.

92. Selden, N.R., Anderson, V.C., McCartney, S., Origitano, T.C., Burchiel, K.J., Barbaro, N.M. (2013). Society of Neurological Surgeons boot camp courses: knowledge retention and relevance of hands-on learning after 6 months of postgraduate year 1 training, J Neurosurg, 119, 3, pp. 796-802.

93. Sheahan, M.G., Duran, C., Bismuth, J. (2015). National Simulation-Based Training of Fellows: The Vascular Surgery Example, Surg Clin North Am, 95, 4, pp. 781-90.

94. Somasundram, K., Spence, H., Colquhoun, A.J., Mcilhenny, C., Biyani, C.S., Jain, S. (2018). Simulation in urology to train non-technical skills in ward rounds, BJU Int, 122, 4, pp. 705-712.

95. Sonnadara, R.R., Van Vliet, A., Safir, O., Alman, B., Ferguson, P., Kraemer, W., Reznick, R. (2011). Orthopedic boot camp: examining the effectiveness of an intensive surgical skills course, Surgery, 149, 6, pp. 745-9.

96. Sonnadara, R.R., Garbedian, S., Safir, O., Nousiainen, M., Alman, B., Ferguson, P., Kraemer, W., Reznick, R. (2012). Orthopaedic Boot Camp II: examining the retention rates of an intensive surgical skills course, Surgery, 151, 6, pp.803-7.

97. Sonnadara, R.R., Garbedian, S., Safir, O., Mui, C., Mironova, P., Nousiainen, M., Ferguson, P., Alman, B., Kraemer, W., Reznick, R. (2013). Toronto orthopaedic boot camp III: examining the efficacy of student-regulated learning during an intensive, laboratory-based surgical skills course, Surgery, 154, 1, pp.29-33. 
98. Starr, M., Sawyer, T., Jones, M., Batra, M., McPhillips, H. (2017). A Simulation-based Quality Improvement Approach to Improve Pediatric Resident Competency with Required Procedures, Cureus, 9, 6, p. e1307. doi: 10.7759/cureus.1307.

99. Strom, S.L., Anderson, C.L., Yang, L., Canales, C., Amin, A., Lotfipour, S., McCoy, C.E., Osborn, M.B., Langdorf, M.I. (2015). Correlation of Simulation Examination to Written Test Scores for Advanced Cardiac Life Support Testing: Prospective Cohort Study, West J Emerg Med, 16, 6, pp. 907-12.

100.Ting, D.S., Lee, J.C., Loo, B.K., Baisa, K., Koo, W.H., Cook, S., Lim, B.L. (2016). A nationwide, resident-led teaching programme for medical students in Singapore: SingHealth Student Internship Programme Bootcamp, Singapore Med J, 57, 5, pp. 233-7.

101.Ulbrich, T.R., Boyle, J.A. (2017). Preparing students for residency interviews through a residency interview boot camp, Curr Pharm Teach Learn, 9, 4, pp. 671-682.

102. Vergara, V.M., Panaiotis, Kingsley. D., Alverson, D.C., Godsmith, T., Xia, S., Caudell, T.P. (2009). The use of virtual reality simulation of head trauma in a surgical boot camp, Stud Health Technol Inform, 142, pp.395-7.

103.Walton, J.L., Dunn, D.K., Haines, N.Y., Heisler, I., Bigham, M.T., Volsko, T.A. (2018). Ventilator Boot Camp Improves the Knowledge and Skills Associated With Mechanical Ventilator Use During Interfacility Transport of Intubated
Pediatric Patients, Respir Care, 63, 4, pp. 417423.

104.Wancata, L.M., Morgan, H., Sandhu, G., Santen, S., Hughes, D.T. (2017). Using the ACMGE Milestones as a Handover Tool From Medical School to Surgery Residency, J Surg Educ, 74, 3, pp. 519-529.

105.Wayne, D.B., Cohen, E.R., Singer, B.D., Moazed, F., Barsuk, J.H., Lyons, E.A., Butter, J., McGaghie,. W.C. (2014). Progress toward improving medical school graduates' skills via a "boot camp" curriculum, Simul Healthc, 9, 1, pp. 33-9.

106.Weis, J.J., Farr, D., Abdelfattah, K.R., Hogg, D., Scott, D.J. (2018). A proficiency-based surgical boot camp May not provide trainees with a durable foundation in fundamental surgical skills, Am J Surg, S0002-9610, 18, pp. 30534-8.

107.Yee, J., Fuenning, C., George, R., Hejal, R., Haines, N., Dunn, D., Gothard, M.D., Ahmed, R.A. (2016). Mechanical Ventilation Boot Camp: A Simulation-Based Pilot Study, Crit Care Res Pract, 2016, p. 4670672. doi: 10.1155/2016/4670672. Epub 2016 Feb 1.

108. Yee, J., Benner, A., Hammond, J., Malone, B., Fuenning, C., George, R., Ahmed, R.A. (2018). Mechanical Ventilation Boot Camp Curriculum, J Vis Exp, 12, p.133 doi: 10.3791/57303.

109.Yeh, D.H., Fung, K., Malekzadeh, S. (2017). Boot Camps: Preparing for Residency, Otolaryngol Clin North Am, 50, 5, pp. 10031013. 